Journal of Applied Analysis

Vol. 6, No. 1 (2000), pp. 1-24

\title{
FEYNMAN'S PATH INTEGRALS AND HENSTOCK'S NON-ABSOLUTE INTEGRATION
}

\author{
P. MULDOWNEY
}

Received August 14, 1998

\begin{abstract}
R. Feynman formulated quantum mechanics in terms of integrals over spaces of paths (Feynman path integrals). But the absolute value of Feynman's integrand is not integrable. And his integrand does not generate a measure. So Lebesgue integration theory could not be used by Feynman. To establish the equivalence of his theory with the traditional formulation of quantum mechanics, Feynman gave an argument that his path integral satisfies Schrödinger's equation. This paper gives a proof of this part of Feynman's theory. To justify Feynman's and other investigators' use of the language and concepts of integration and probability theory, and to justify taking limits under the integral sign in Feynman's integral, we use R. Henstock's approach to non-absolute integration, which does not require the measure concept, and for which the absolute value of the integrand need not be integrable.
\end{abstract}

\section{Introduction}

Feynman describes his paper as follows [2]:

1991 Mathematics Subject Classification. 28C20, 46G12, 58C35, 81C35.

Key words and phrases. Henstock integral, Feynman path integral, Brownian motion, Feynman-Kac formula, limits under the integral sign.

ISSN 1425-6908 (c) Heldermann Verlag. 
"Non-relativistic quantum mechanics is formulated here in a different way. It is, however, mathematically equivalent to the familiar formulation. In quantum mechanics the probability of an event which can happen in several different ways is the absolute square of a sum of complex contributions, one from each alternative way. The probability that a particle will be found to have a path $x(t)$ lying somewhere within a region of space time is the square of a sum of contributions, one from each path in the region. The contribution from a single path is postulated to be an exponential whose (imaginary) phase is the classical action (in units of $\hbar$ ) for the path in question. The total contribution from all paths reaching $x, t$ from the past is the wave function $\psi(x, t)$. This is shown to satisfy Schroedinger's equation."

Feynman's demonstration of Schrödinger's equation involves taking limits of the sum over a space of paths of a functional of the action for each path. He did not provide proofs.

This paper provides appropriate definitions and proofs. Aspects of some of the probabilistic features of Feynman's thesis are discussed in Muldowney [13].

We modify slightly the notation used by Feynman. Feynman argues that the probability that an "ideal measurement" will determine that a particle moving in one dimension has a path $x$ contained in

$$
J=\left\{x: x\left(\tau^{\prime}\right)=\xi^{\prime}, u_{j} \leq x_{j}<v_{j}, x(\tau)=\xi\right\},
$$

where $\tau_{0}=\tau^{\prime}, \varepsilon=n^{-1}\left(\tau-\tau^{\prime}\right), \tau_{j}=\tau_{j-1}+\varepsilon$ for $j=1,2, \ldots, n$, and $x_{j}$ denotes the position of the particle at time $\tau_{j}$, is the square of the absolute value of

$$
\begin{array}{r}
\int_{u_{1}}^{v_{1}} \cdots \int_{u_{n-1}}^{v_{n-1}} \exp \sum_{j=1}^{n}\left[\frac{\iota \varepsilon}{\hbar}\left\{\frac{m}{2}\left(\frac{x_{j}-x_{j-1}}{\varepsilon}\right)^{2}-V\left(x_{j}\right)\right\}\right] \\
\cdots \frac{d x_{1}}{A} \\
\cdots \frac{d x_{n-1}}{A} .
\end{array}
$$

This corresponds to Equation (23) of Feynman's paper [2, p. 375]. ( $\iota=\sqrt{-1}$, and $A$ is a normalising constant.) Feynman calls (1) the probability amplitude for $J$.

Feynman then argues, in effect, that the state function $\psi(\xi, \tau)$ of the particle, conditional on displacement $\xi^{\prime}$ at time $\tau^{\prime}$ and displacement $\xi$ at time $\tau$ is

$$
\begin{array}{r}
\lim _{\varepsilon \rightarrow 0} \int_{-\infty}^{\infty} \cdots \int_{-\infty}^{\infty} \exp \sum_{j=1}^{n}\left[\frac{\iota \varepsilon}{\hbar}\left\{\frac{m}{2}\left(\frac{x_{j}-x_{j-1}}{\varepsilon}\right)^{2}-V\left(x_{j}\right)\right\}\right] \frac{d x_{1}}{A} \\
\cdots \frac{d x_{n-1}}{A},
\end{array}
$$


where $A=(2 \pi \varepsilon \iota)^{1 / 2}$. This corresponds to Equation (9) of Feynman [2, p. 371]. The number $n-1$ of partition points $\tau_{1}, \ldots, \tau_{n-1}$ increases as $\varepsilon$ decreases.

On pages 375 and 376, Feynman outlines an argument that $\psi$, defined by this limit, satisfies Schrödinger's equation.

In a footnote on page 371 , Feynman says:

"There are very interesting mathematical problems involved in the attempt to avoid the subdivision and limiting process. Some sort of complex measure is being associated with the space of functions $x(t)$. Finite results can be obtained under unexpected circumstances because the measure is not positive everywhere, but the contributions from most of the paths largely cancel out. These curious mathematical problems are sidestepped by the subdivision process. However, one feels as Cavalieri must have felt calculating the volume of a pyramid before the invention of calculus."

In a letter to the author, dated March 30 1982, Feynman wrote:

"I didn't know how to define the measure of the space of paths that was independent of the nature of what I was integrating. So I had to divide the interval of $t$ into steps of size $\varepsilon$ and define the measure as the product over all $\varepsilon$ of $d x_{1} / A(\varepsilon)$ where

$$
A(\varepsilon)=\sqrt{\frac{2 \pi \iota \hbar \varepsilon}{m}}
$$

for the one-dimensional example where the kinetic energy is $m \dot{x}^{2} / 2$. ( $x_{i}$ is the coordinate at the $k$ th point, e.g., at time = original time plus ke.) Only if $A$ has this form will the limit as $\varepsilon \rightarrow 0$ exist. This is all necessary because paths which are not differentiable are the important paths."

Problems associated with Feynman's subdivision process include the following.

1. Is the multi-dimensional integral in (2) above an approximation to an integral over the infinite-dimensional space of paths whose end points are $\left(\xi^{\prime}, \tau^{\prime}\right)$ and $(\xi, \tau)$ ?

2. And, for such infinite-dimensional integrals, is there a suitable theorem on taking limits under the integral sign which will enable us to deduce Schrödinger's equation?

If the answer to each question is yes, it may be possible to accomplish Feynman's programe in the manner set out in his paper.

As Kac [8] showed, the analogous problem with $\iota$ replaced by -1 in Feynman's expressions for the probability amplitude and state function, (1) and (2), is solvable by Lebesgue integration. ${ }^{1}$ But the solution in this case

\footnotetext{
${ }^{1}$ Suppose that $\iota$ is replaced by -1 . A semi-algebra of cylinder sets, and a pre-measure corresponding to Feynman's probability amplitude 1 defined on them, are extended to a $\sigma$-algebra and measure (the Wiener measure), respectively.
} 
depends on the absolute integrability of the Wiener pre-measure, and if we replace the expression

$$
\exp \left[\frac{\iota \varepsilon}{h}\left\{\frac{m}{2}\left(\frac{x_{j}-x_{j-1}}{\varepsilon}\right)^{2}-V\left(x_{j}\right)\right\}\right]
$$

in the Feynman pre-measure (1) by its absolute value, all sums diverge.

A non-absolute method of integration is required in order to allow the cancellation effects described by Feynman to come into play. So, instead of Lebesgue integration, we use the non-absolute integral of Henstock.

\section{The Henstock integral}

Instead of a fixed $\varepsilon=n^{-1}\left(\tau-\tau^{\prime}\right)$, replace $\tau_{1}, \ldots, \tau_{n-1}$ by arbitrary partition points, $\tau^{\prime}=t_{0}<t_{1}<\ldots<t_{n}=\tau$, and let $J$ be a cylinder set in $\mathbf{R}^{\tau^{\prime}, \tau[}$

$$
J=\left\{x: x\left(\tau^{\prime}\right)=\xi^{\prime}, u_{j} \leq x\left(t_{j}\right)<v_{j}, j=1,2, \ldots, n-1, x(\tau)=\xi\right\} .
$$

Let $x_{j}$ denote $x\left(t_{j}\right)$, and let

$$
\int_{u_{1}}^{v_{1}} \ldots \int_{u_{n-1}}^{v_{n-1}} \exp \left(\lambda \sum_{j=1}^{n} \frac{\left(x_{j}-x_{j-1}\right)^{2}}{2\left(t_{j}-t_{j-1}\right)}\right) \prod_{j=1}^{n}\left(\frac{2 \pi}{-\lambda}\left(t_{j}-t_{j-1}\right)\right)^{-1 / 2} d x_{1}
$$

be a pre-measure defined on cylinder sets.

As discussed above, if $\lambda=-1$, this pre-measure can be extended to a measure on the measure space generated by the cylinder sets in $\mathbf{R}^{\tau^{\prime}, \tau \text {. }}$. This is the Wiener measure, which is, in turn, equivalent in a certain sense to a measure $w$ which is concentrated on the subset of continuous functions

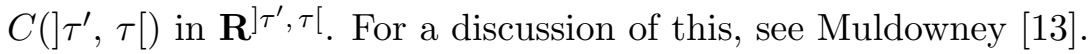

So

$$
\phi(\xi, \tau):=\int_{C(] \tau^{\prime}, \tau[)}\left(\int_{\tau^{\prime}}^{\tau} \exp (-V(x(t)) d t) d w\right.
$$

is defined as a Lebesgue integral with respect to the measure $w$.

Kac [8] showed that a programme analogous to Feynman's can be accomplished in this case, leading to a diffusion equation for $\phi$ analogous to Schrödinger's.

But Lebesgue integration requires that the absolute value of the integrand be integrable. And if $\lambda=\iota:=\sqrt{-1}$, then the expression inside the integral sign in the above pre-measure takes an absolute value of 1 , so (3) is not integrable as $t_{j}-t_{j-1}$ tends to zero. Taking the absolute value of the 
integrand in (3) causes the loss of the cancellation effects which are present in sums containing the expression as it stands.

Therefore we approach the problem using the non-absolute integral of Henstock, also known as the generalised Riemann integral.

We give a definition of the generalised Riemann integral in $\mathbf{R}$. If $I$ is a bounded real interval $[u, v[$ let $|I|$ be $v-u$. If $I$ is an unbounded interval, let $|I|$ be 0 . Let $\delta(x)$ be a positive function defined for $x \in \overline{\mathbf{R}}=\mathbf{R} \cup\{-\infty, \infty\}$. The function $\delta$ is called a gauge. Given $x \in[-\infty, \infty]$, and $-\infty<u<v<$ $\infty$, we say that the real interval $I$ is attached to $x$ if

$$
I= \begin{cases}{[u, v[} & \text { and } x=u \text { or } v, \text { or } \\ ]-\infty, v[\text { and } x=-\infty, \text { or } \\ {[u, \infty[\text { and } x=\infty,}\end{cases}
$$

Taking each of these three cases in turn, if $I$ is attached to $x$ we say the point-interval pair $(x, I)$ is $\delta$-fine if

$$
v-u<\delta(x) ; \text { or } v<-\frac{1}{\delta(x)} ; \text { or } u>\frac{1}{\delta(x)}, \text { respectively. }
$$

A finite collection of attached point-interval pairs

$$
\mathcal{E}=\{(x, I)\}=\left\{\left(x^{(1)}, I^{(1)}\right), \ldots,\left(x^{(m)}, I^{(m)}\right)\right\}
$$

is a division of $\mathbf{R}$ if the finite set of intervals $I^{(j)}$ form a non-intersecting cover for $\mathbf{R}$. The division $\mathcal{E}_{\delta}$ is $\delta$-fine if each $(x, I)$ in $\mathcal{E}_{\delta}$ is $\delta$-fine. Note that as $\delta(x)$ decreases, the number of partition points of $\mathbf{R}$ increases.

Now suppose $h$ is a real- or complex-valued function of point-interval pairs $(x, I)$, with $h(x, I):=0$ if $x=-\infty$ or $\infty$. For example, $h(x, I)$ could be a point function $f(x)$ multiplied by the interval length $|I|=v-u$. Then $h$ is integrable over $\mathbf{R}$ in the generalised Riemann sense, and has integral $\alpha$, if, given $\varepsilon>0$, there exists a gauge $\delta$ such that

$$
\left|\left(\mathcal{E}_{\delta}\right) \sum h(x, I)-\alpha\right|<\varepsilon
$$

for every $\delta$-fine division $\mathcal{E}_{\delta}$ of $\mathbf{R}$. $\left(\mathcal{E}_{\delta}\right) \sum h(x, I)$ denotes the Riemann sum over any $\delta$-fine division $\mathcal{E}_{\delta}$, and $\int_{\mathbf{R}} h(x, I)$ denotes the generalised Riemann integral $\alpha$ of $h$. Note that the generalised Riemann integral over the domain ] $-\infty, \infty[$ does not require use of the Cauchy extension.

Suppose $h$ has the form $f(x) \mu(I)$, and $\mu$ is a measure. Whenever $f$ is finite, real-valued, $\mu$-measurable and Lebesgue integrable over $\mathbf{R}$ with respect to $\mu$, then $f(x) \mu(I)$ is generalised Riemann integrable, and

$$
\int_{\mathbf{R}} f(x) \mu(I)=\int_{-\infty}^{\infty} f(x) d \mu,
$$

where the latter integral is the Lebesgue, and the former is the generalised Riemann integral. The sense of the notation $\int f(x) \mu(I)$ is that the space 
in which the integration is performed is divided into intervals $I$, and the integral is approximated by Riemann sums $\sum f(x) \mu(I)$. (We will frequently use notation of the form $\int f(x) \mu(I)$ to indicate Henstock or generalised Riemann integration, $\int f(x) d \mu$ to indicate Lebesgue integration and $\int f(x) d x$ for ordinary Riemann integration.)

For the Henstock integral in infinite-dimensional spaces, let $B$ be an indexing set with infinitely many elements. We start by considering cylindrical intervals in $\mathbf{R}^{B}$. Let $I(N)$ be any $n$-dimensional interval, or parallelepiped, in $\mathbf{R}^{N}$,

$$
I(N):=I_{b_{1}} \times \ldots \times I_{b_{n}}
$$

where each $I_{b_{j}}$ is a real interval of the form $[u, v),(-\infty, v)$, or $[u,+\infty)$; and $I(N)$ is attached to $x(N):=\left(x\left(b_{1}\right), \ldots, x\left(b_{n}\right)\right)$ if $x\left(b_{j}\right)=u$ or $v,-\infty$, or $\infty$, respectively. Let

$$
I=I[N]:=I(N) \times \mathbf{R}^{B \backslash N} .
$$

In another notation, $I$ is

$$
\left\{x: x \in \mathbf{R}^{B}, x\left(b_{j}\right) \in I_{b_{j}}, 1 \leq j \leq n\right\} .
$$

Let $|I(N)|$ denote $\left|I_{b_{1}}\right| \times \ldots \times\left|I_{b_{n}}\right|$, and let $|I[N]|:=|I(N)| .|I[N]|$ is the volume of the finite dimensional interval $I(N)$ obtained when $I[N]$ is projected into the dimensions $N$ in which the infinite dimensional cylindrical interval is restricted. We write $I[N]$ instead of $I$ whenever we wish to emphasise that $N$ is the set of dimensions in which the cylindrical interval $I$ is restricted.

We say that $I[N]$ is attached to $x$ if $I(N)$ is attached to $x(N)$.

An elementary set $E$ is an interval or a finite union of intervals. $\mathbf{R}^{B}$ is an elementary set.

If $f$ is a functional of $x \in \mathbf{R}^{B}$ and $\mu$ is a volume function defined on cylindrical intervals $I[N]$, we consider Riemann sums

$$
\sum f(x) \mu(I[N])
$$

which will, in some sense, approximate an integral in $\mathbf{R}^{B}$ which we wish to define. Each $I[N]$ is attached to the corresponding $x$ in the Riemann sum. $f(x)$ is zero by definition if $x(N)$ has any infinite component. The cylindrical intervals $I[N]$ in the Riemann sum form a disjoint finite cover for $\mathbf{R}^{B}$. Note that the dimension sets $N$ are variable in the Riemann sum.

More generally, we consider functionals $h(x, I[N])$, and Riemann sums

$$
\sum h(x, I[N])
$$

approximating the integral $\int_{\mathbf{R}^{B}} h$, which we have yet to define.

To obtain convergence of the Riemann sums, we must examine sequences of Riemann sums in which the cylindrical intervals $I[N]$ "shrink" according 
to some rule. Suppose the cylindrical interval $J[M] \subseteq I[N]$ has $M \supseteq$ $N$ and each restricted edge of $J[M]$ is no greater than the corresponding edge of $I[N]$. Then $J[M]$ is a proper subset of $I[N]$ if $M$ contains $N$ as a proper subset, or each restricted edge of $J[M]$ is strictly shorter than the corresponding edge of $I[N]$, or both.

Guided by this, we obtain a "shrinking rule" or gauge for the Riemann sums as follows. For each $x \in \overline{\mathbf{R}^{B}}$ (that is, $\mathbf{R}^{B}$ with points at infinity adjoined), let $\delta(x)$ be a positive number. If $B$ is uncountable, let $A$ be a countable subset of $B$. If $B$ is countable take $A=B$. Let $\mathbf{L}_{A}$ be the family of finite subsets of $A$, and, for each $x \in \overline{\mathbf{R}^{B}}$, let $L_{A}(x)$ be a member of $\mathbf{L}_{A}$. So

$$
\begin{aligned}
\delta & : \quad \overline{\mathbf{R}^{B}} \longmapsto \mathbf{R}^{+}, \\
L_{A} & : \quad \overline{\mathbf{R}^{B}} \longmapsto \mathbf{L}_{A} .
\end{aligned}
$$

A gauge $\gamma$ is defined as

$$
\gamma:=\left(\delta, L_{A}\right)
$$

Suppose $I[N]$ is attached to $x$. We say that $(x, I[N])$ is $\gamma$-fine if $N$ contains $L(x)$, and if, taking $\delta(x(b))=\delta(x)$, the one-dimensional intervals $I_{b}$ in $\mathbf{R}$ are $\delta$-fine for each $b \in N$.

Thus, any gauge $\gamma$ depends on a choice of positive numbers $\delta(x)$, one for each $x \in \overline{R^{B}}$, a choice of a countable dimension set $A \subset B$, and a choice of finite subsets $L_{A}(x)$ of $A$, one for each $x \in \overline{R^{B}}$.

(If $B$ were a finite set with $n$ elements, then we would take $L_{A}(x)=A=$ $B$ for every $x$, and likewise $N=B$, so that every cylindrical interval $I[N]$ and every parallelepiped $I(N)$ is just the usual rectangular interval in the fixed $n$-dimensional space $\mathbf{R}^{n}$; and a gauge $\gamma$ reduces to the familiar gauge $\delta$ defined at each point of $\overline{\mathbf{R}^{n}}$. For more details of this, see Muldowney $[10$, pp. $8-10]$.

The role of the countable set $A$ in the definition of a gauge is to ensure that $\mathbf{R}^{B}$ (or any other elementary set $E$ ) can be covered by a finite set of mutually exclusive $\gamma$-fine cylindrical intervals $I[N]$. See Muldowney [12] for a proof.

So, given a gauge $\gamma$ we can find a finite collection of attached pointinterval pairs

$$
\mathcal{E}_{\gamma}=\{(x, I[N])\}
$$

in which each $(x, I[N])$ is $\gamma$-fine, and the cylindrical intervals $I[N]$ are mutually exclusive and have union $\mathbf{R}^{B}$ (or $E$ ). We call $\mathcal{E}_{\gamma}$ a $\gamma$-fine division of $\mathbf{R}^{B}$ (or $E$ ) and denote the Riemann sum by $\left(\mathcal{E}_{\gamma}\right) \sum h(x, N, I[N])$.

Taking $h$ to be zero by definition if $x(N)$ is a point at infinity; that is, if $x(N)$ has an infinite component, we say that $h$ is integrable in $\mathbf{R}^{B}$ with generalised Riemann integral $\alpha$ if given $\varepsilon>0$ we can choose a gauge $\gamma$ so 
that, for every $\gamma$-fine division $\mathcal{E}_{\gamma}$ of $\mathbf{R}^{B}$, we have

$$
\left|\left(\mathcal{E}_{\gamma}\right) \sum h(x, N, I[N])-\alpha\right|<\varepsilon,
$$

and we write

$$
\alpha=\int_{\mathbf{R}^{B}} h(x, N, I), \text { or just } \int_{\mathbf{R}^{B}} h .
$$

Likewise for the integrability of $h$ in an elementary set $E$, and $\int_{E} h$. If $X$ is a subset of $\mathbf{R}^{B}$ which is not an elementary set, let $\mathbf{1}_{X}$ denote the indicator function of $X$ and define $\int_{X} h(x, N, I)$ to be

$$
\int_{\mathbf{R}^{B}} \mathbf{1}_{X}(x) h(x, N, I)
$$

if $\mathbf{1}_{X}(x) h(x, N, I)$ is integrable.

Note that the functions (or functionals) $h$ may depend explicitly on the dimension sets $N$, as in (3), which is why we use the functional form $h(x, N, I)$ instead of $h(x, I)$. The integrability of such functions is demonstrated in Muldowney [10, Chapters 3 and 4]. For proofs of Fubini's theorem, the dominated convergence theorem and other properties of the integral, see Muldowney [10, Chapter 2].

Henstock formulated the theory of integration in function spaces in [4, pp. 210-249], [5] and [6].

\section{Henstock's criteria}

We state and prove Henstock's necessary and sufficient conditions for taking limits under the integral sign.

The problems are as follows. If $h_{n}$ is integrable and $h_{n} \rightarrow h$ as $n \rightarrow \infty$, then

1. When is $h$ integrable?

2. When does $\lim _{n \rightarrow \infty} \int h_{n}$ exist?

3. When does $\int h=\lim _{n \rightarrow \infty} \int h_{n}$ ?

Let $E$ be an elementary set in $\mathbf{R}^{B}$. $E$ may be unbounded in some or all dimensions, for instance, if $E=\mathbf{R}^{B}$. So, when we say that $h_{n} \rightarrow h$, it is not sufficient that $n>n_{0}$ implies $\left|h_{n}(x, N, I)-h(x, N, I)\right|<\varepsilon$ for each $x, N, I$.

Instead we need the following. $h_{m}$ converges boundedly to $h$ if there exist a positive function $\beta(x, N, I)$, a gauge $\gamma_{0}$ and integers $m_{0}=m_{0}(x, N, I)$ defined for each $\gamma_{0}$-fine $(x, I[N])$ so that, firstly, $\left(\mathcal{E}_{\gamma_{0}}\right) \sum \beta(x, N, I)$ is bounded 
for all $\gamma_{0}$-fine divisions $\mathcal{E}_{\gamma_{0}}$ of $E$ and, secondly, $m>m_{0}(x, N, I)$ implies $^{2}$

$$
\left|h_{n}(x, N, I)-h(x, N, I)\right|<\varepsilon \beta(x, N, I)
$$

for all $\gamma_{0}$-fine $(x, I[N])$.

The following proposition gives Henstock's criteria for taking limits under the integral sign. The statement and proof are adapted from Henstock [7, pp. 120-125]. $B(a, b)$ denotes a ball with centre $a$ and radius $b$.

Proposition 1 (Henstock's criteria). Suppose $h_{n}(x, N, I)$ converges boundedly to $h(x, N, I)$.

1. $h$ is integrable in $E$ if and only if there exist a ball $B_{1}$ of arbitrarily small radius, and, correspondingly, a gauge $\gamma$, and integers $p=$ $p(x, N, I)$ so that $m=m(x, N, I)>p(x, N, I)$ and for each $\mathcal{E}_{\gamma}$

$$
\left(\mathcal{E}_{\gamma}\right) \sum h_{m(x, N, I)}(x, N, I) \in B_{1} .
$$

2. $\lim _{n \rightarrow \infty} \int_{E} h_{n}$ exists if and only if there exist a ball $B_{2}$ of arbitrarily small radius, a corresponding integer $n_{0}$ and, for each $n>n_{0}$, a gauge $\gamma_{n}$ so that for each $\mathcal{E}_{\gamma_{n}}$

$$
\left(\mathcal{E}_{\gamma_{n}}\right) \sum h_{n}(x, N, I) \in B_{2} .
$$

3. Given the existence of $\int_{E} h$ and $\lim _{n \rightarrow \infty} \int_{E} h_{n}$, the two are equal if and only if

$$
B_{1} \cap B_{2} \neq \emptyset \text {. }
$$

(In other words, if and only if $B_{1}$ and $B_{2}$ are concentric.)

Proof. 1. If $h$ is integrable, with $\int_{E} h=a$, then let $\varepsilon>0$ be given, take $\gamma=\gamma_{0}$, take $p(x, N, I)=m_{0}(x, N, I)$, and let $K$ be an upper bound for $\left(\mathcal{E}_{\gamma_{0}}\right) \sum \beta(x, N, I)$ for all $\mathcal{E}_{\gamma_{0}}$, so that

$$
\left(\mathcal{E}_{\gamma_{0}}\right) \sum h_{m(x, N, I)}(x, N, I) \in B(a, K \varepsilon) .
$$

Conversely, if there exist $B_{1}, \gamma$ and $p(x, N, I)$ so that

$$
\left(\mathcal{E}_{\gamma}\right) \sum h_{m(x, N, I)}(x, N, I) \in B_{1},
$$

then with $K$ as before, take $\gamma^{\prime}=\gamma_{0} \wedge \gamma$ and

$$
m(x, N, I) \geq \max \left\{p(x, N, I), m_{0}(x, N, I)\right\}
$$

for $(x, I[N]) \gamma^{\prime}$-fine, so that for each $\mathcal{E}_{\gamma^{\prime}}$,

$$
\left(\mathcal{E}_{\gamma^{\prime}}\right) \sum h(x, N, I) \in B_{1}+B(0, K \varepsilon),
$$

\footnotetext{
${ }^{2}$ If $h_{n}=f_{n}(x, N) p(I)$, and if $p(I)$ is $\mathrm{VBG}^{*}$ (has generalised bounded variation), then bounded convergence of $h_{n}$ is equivalent to pointwise convergence of $f_{n}(x, N)$, as we shall see.
} 
giving the integrability of $h$.

2. If $\lim _{n \rightarrow \infty} \int_{E} h_{n},=b$, exists, then there exists $n_{0}$ so that $n>n_{0}$ implies $\int_{E} h_{n} \in B(b, \varepsilon)$. In other words, there exists $\gamma_{n}$ so that for all $\mathcal{E}_{\gamma_{n}}$,

$$
\left(\mathcal{E}_{\gamma_{n}}\right) \sum h_{n}(x, N, I) \in B(b, 2 \varepsilon) .
$$

Conversely, suppose there exist $B_{2}, n_{0}$ and $\gamma_{n}$, with $n>n_{0}$, so that $\left(\mathcal{E}_{\gamma_{n}}\right) \sum h_{n} \in B_{2}$. Since each $h_{n}$ is integrable there exist gauges $\gamma_{n}^{\prime}$ so that for all $\mathcal{E}_{\gamma_{n}^{\prime}}\left|\left(\mathcal{E}_{\gamma_{n}^{\prime}}\right) \sum h_{n}-\int_{E} h_{n}\right|<\varepsilon$. Now take $\gamma_{n}^{\prime \prime} \prec \gamma_{n} \wedge \gamma_{n}^{\prime}$ and take $\gamma_{n}^{\prime \prime}$-fine divisions. Then, for $n>n_{0}, \int_{E} h_{n} \in B_{2}+B(0, \varepsilon)$, so $\lim _{n \rightarrow \infty} \int_{E} h_{n}$ exists.

3. If $\varepsilon$ is an arbitrary positive number then, given the existence of $a=$ $\int_{E} h$ and $b=\lim _{n \rightarrow \infty} \int_{E} h_{n}$, we have $a=b$ if and only if $B_{1}=B(a, K \varepsilon)$ and $B_{2}=B(b, 2 \varepsilon)$ have non-empty intersection.

\section{The Feynman integral}

The first stage in the construction of the Lebesgue integral in function spaces (see [13]) is to consider cylinder sets on which a pre-measure is defined. In the second stage the cylinder sets and pre-measure generate, respectively, a $\sigma$-algebra of sets in the function space, and a measure defined on these which is used to integrate measurable functions of points in the function space.

In the preceding sections, we have described the corresponding construction of Henstock's generalised Riemann integral. The definition in this case needs only the machinery of the first stage of the Lebesgue definition, that is, cylinder sets and the pre-measures or volume functions defined on them. It is possible at this stage to restrict our discussion to integrands which conform to the traditional structure of point function multiplied by set function, $f(x) \times \mu(I)$. The analysis of Feynman integrals which we are engaged upon can be conducted in these terms. But it is more comprehensive and, arguably, simpler, to treat the integrand, not as a product, but as a joint function $h(x, I)$ (or functional) of points $x$ in the function space and of

cylinder sets $I$. The latter approach also has the psychological benefit of reducing the measure theoretic "feel" of the subject, and this is an advantage in the Feynman problem where measure theory has little to contribute.

Our starting point in Feynman integration is the expression (1) above

$$
\int_{u_{1}}^{v_{1}} \cdots \int_{u_{n-1}}^{v_{n-1}} \exp \sum_{j=1}^{n}\left[\frac{\iota \varepsilon}{\hbar}\left\{\frac{m}{2}\left(\frac{x_{j}-x_{j-1}}{\varepsilon}\right)^{2}-V\left(x_{j}\right)\right\}\right] \frac{d x_{1}}{A} \cdots \frac{d x_{n-1}}{A},
$$


which, taking $V=0$, we have modified to the form (3)

$$
\begin{array}{r}
\int_{u_{1}}^{v_{1}} \cdots \int_{u_{n-1}}^{v_{n-1}} \exp \left(\lambda \sum_{j=1}^{n} \frac{\left(x_{j}-x_{j-1}\right)^{2}}{2\left(t_{j}-t_{j-1}\right)}\right) \prod_{j=1}^{n}\left(\frac{2 \pi}{-\lambda}\left(t_{j}-t_{j-1}\right)\right)^{-1 / 2} d x_{1} \\
\ldots d x_{n-1} .
\end{array}
$$

This is to be read as a volume function defined on cylinder sets in $\mathbf{R}^{] \tau^{\prime}, \tau[\text {, but }}$ can also be interpreted as a volume function defined on the finite dimensional space $\mathbf{R}^{N}$, with $x(N)=\left(x_{1}, \ldots, x_{n-1}\right)$.

With $\lambda=a+\iota b, a \leq 0, b \geq 0, \lambda \neq 0$, and $V$ a real-valued function of a single real variable, let $g_{V}(x, N)$ denote

$$
\exp \left(\frac{\lambda}{2} \sum_{j=1}^{n} \frac{\left(x_{j}-x_{j-1}\right)^{2}}{t_{j}-t_{j-1}}-V\left(x_{j-1}\right)\left(t_{j}-t_{j-1}\right)\right) \prod_{j=1}^{n}\left(\frac{2 \pi}{-\lambda}\left(t_{j}-t_{j-1}\right)\right)^{-1 / 2}
$$

so, in the free particle case, with $V$ identically zero,

$$
g_{0}(x, N)=\exp \left(\frac{\lambda}{2} \sum_{j=1}^{n} \frac{\left(x_{j}-x_{j-1}\right)^{2}}{t_{j}-t_{j-1}}\right) \prod_{j=1}^{n}\left(\frac{2 \pi}{-\lambda}\left(t_{j}-t_{j-1}\right)\right)^{-1 / 2} .
$$

The above expressions also define the functions $g_{V}(x(N), N), g_{0}(x(N), N)$ on the finite dimensional space $\mathbf{R}^{N}$, where $N$ is now taken to be a fixed set of dimensions. Let

$$
g_{V}(x, N, I):=g_{V}(x, N)|I[N]|, g_{V}(x(N), I(N)):=g_{V}(x(N), I(N))|I(N)| .
$$

The latter is the finite dimensional version of the former. Each of the two functions takes the same complex values.

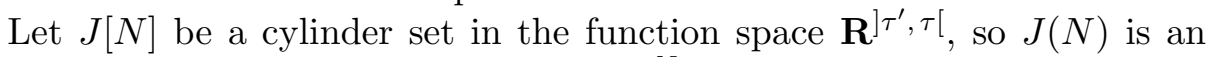
interval in the finite dimensional space $\mathbf{R}^{N}$. Let

$$
G_{V}(J(N)):=\int_{J(N)} g_{V}(x(N), I(N)),
$$

where $N$ is fixed and the integral is finite dimensional, and is approximated by Riemann sums in which $J(N)$ is partitioned by sub-intervals $I(N)$. As a finite dimensional expression with fixed $N, g_{V}$ is integrable, for instance, if $V$ is continuous. Now let

$$
G_{V}(J[N]):=G_{V}(J(N)),
$$

so $G_{V}$ is now defined for infinite dimensional cylinder sets as well as finite dimensional intervals. An obvious corollary of Muldowney [10, Proposition 68 , p. 84$]$ is that, taking $M$ to be a fixed dimension set and $J[M]$ a fixed cylinder set,

$$
G_{0}(J[M])=\int_{J[M]} g_{0}(x, N, I),
$$


where the latter integral is infinite dimensional and is approximated by Riemann sums in which $J[M]$ is partitioned into subintervals $I[N]$ with $N \supseteq M$. When $V$ is not identically zero, the relationship between $G_{V}(J[M])$ and $\int_{J[M]} g_{V}(x, N, I)$ is less simple. Sometimes we want to integrate $G_{V}$. In such situations we require $G_{V}$ to be defined for each associated $x, N, I$, so we take

$$
G_{V}(x, N, I)=G_{V}(I[N]) .
$$

Note that $G_{V}(J)$ corresponds to Feynman's probability amplitude (1) above, with the $\tau_{j}$ 's replaced by $t_{j}$ 's, with the fixed $\varepsilon=\tau_{j}-\tau_{j-1}$ replaced by variable $\varepsilon_{j}=t_{j}-t_{j-1}$, and with units chosen so that $m=\hbar=1$. $G_{0}(J)$ is $(3)$ in an alternative notation. We turn now to an interpretation of the state function $\psi(\xi, \tau)$ corresponding to Feynman's expression (2). Let

$$
v(x, N):=\exp \left(\lambda \sum_{j=1}^{n} V\left(x_{j-1}\right)\left(t_{j}-t_{j-1}\right)\right) .
$$

Assume $V$ is a continuous function of a single real variable. Let $C$ denote the set of continuous $x$ in $\mathbf{R}^{] \tau^{\prime}, \tau[}$ and let $D$ denote $\mathbf{R}^{] \tau^{\prime}, \tau[} \backslash C$. Let

$$
v(x):= \begin{cases}\exp \left(\lambda \int_{\tau^{\prime}}^{\tau} V(x(t)) d t\right) & \text { if } x \in C \\ 0 & \text { if } x \in D\end{cases}
$$

Let $v(x(N)):=v(x, N)$ whenever we need a finite dimensional version of $v$. Let $m=2^{q}$ and let $\tau_{0}=\tau^{\prime}, \tau_{j}=\tau_{j-1}+2^{-q}\left(\tau-\tau^{\prime}\right)$ for $1 \leq j \leq m-1$ so $\tau_{m}=\tau$. Let $M$ denote the fixed set of dimensions $\left\{\tau_{1}, \ldots, \tau_{m-1}\right\}$ and let

$$
v(x(M))=v(x ; M)=\exp \left(\lambda \sum_{j=1}^{m} V\left(x\left(\tau_{j-1}\right)\right)\left(\tau_{j}-\tau_{j-1}\right)\right) .
$$

Let

$$
v_{m}(x):= \begin{cases}v(x ; M) & \text { if } x \in C \\ 0 & \text { if } x \in D\end{cases}
$$

For $x \in C, v_{m}(x) \rightarrow v(x)$ as $m \rightarrow \infty$.

Let $\psi_{V}(\xi, \tau)$ denote the infinite dimensional integral of $v(x, N) G_{0}(I)$ whenever it exists;

$$
\psi_{V}(\xi, \tau)=\int_{\mathbf{R}^{] \tau^{\prime}, \tau[}} v(x \cdot N) G_{0}(I) .
$$

The integrability of $v(x, N) G_{0}(I)$ is discussed in Muldowney [10, Chapter 4, pp. 77-88]. By Muldowney ([10, Proposition 60, p. 79]), each of $v(x, N) G_{0}(I), G_{V}(x, N, I), g_{V}(x, N)|I|$ is integrable in $\mathbf{R}^{] \tau^{\prime}, \tau[}$ whenever any 
one of them is, and then we have

$\psi_{V}(\xi, \tau)=\int_{\mathbf{R}^{] \tau^{\prime}}, \tau[} v(x, N) G_{0}(I)=\int_{\mathbf{R}^{] \tau^{\prime}}, \tau[} G_{V}(x, N, I)=\int_{\mathbf{R}^{] \tau^{\prime}}, \tau[} g_{V}(x, N)|I|$.

It is shown in Muldowney [10, Proposition 68, p. 84, and Proposition 60, p. 79], that $G_{0}(x, N, I)$ and $g_{0}(x, N)|I|$ are integrable and

$$
\int_{\mathbf{R}^{\left.\mid \tau^{\prime}, \tau\right]}} G_{0}(x, N, I)=\int_{\mathbf{R}^{] \tau^{\prime}, \tau[}} g_{0}(x, N)|I|=\left(\frac{\pi}{-\lambda}\left(\tau-\tau^{\prime}\right)\right)^{-1 / 2} \exp \frac{\lambda\left(\xi-\xi^{\prime}\right)^{2}}{\tau-\tau^{\prime}},
$$

which is, therefore, $\psi_{0}(\xi, \tau)$. Thus Henstock's integral gives the correct form of the quantum mechanical state function in the case of a single free particle moving in one dimension ( $V$ identically 0 ), and so justifies the use of $\psi$ notation in this case. A fuller justification is given later.

As before, take $\mathbf{1}_{X}$ to be the indicator function of any set $X$, so

$$
\mathbf{1}_{X}(x)= \begin{cases}1 & \text { if } x \in X \\ 0 & \text { otherwise }\end{cases}
$$

Let $C_{M}$ denote the set of $x$ in $\mathbf{R}^{] \tau^{\prime}, \tau[}$ which are continuous at each $t$ in $M$ so $\mathbf{1}_{C_{M}}$ is the indicator functional of $C_{M}$.

It is shown in Muldowney ([10, Proposition 77, p. 91]), that $\mathbf{R}^{] \tau^{\prime}, \tau[} \backslash C_{M}$ is a null set relative to $G_{0}$, and

$$
\begin{aligned}
\psi_{V}(\xi, \tau) & =\int_{\mathbf{R}] \tau^{\prime}, \tau \mid} g_{V}(x, N)|I| \mathbf{1}_{C_{M}}(x) \\
& =\int_{\mathbf{R}^{\mid \tau^{\prime}, \tau[}} v(x, N) G_{0}(I) \mathbf{1}_{C_{M}}(x)
\end{aligned}
$$

whenever $\psi_{V}(\xi, \tau)$ exists. See also Muldowney [11, pp. 131-135].

$C$ is the set of continuous $x$ in $\mathbf{R}^{] \tau^{\prime}, \tau[}$ and is a subset of $C_{M}$ which is, in a sense, much smaller than $C_{M}$. Nonetheless, it is possible to define a cylinder function $F_{0}$ which is, in the terminology of probability theory, a continuous modification of $G_{0}$, so that

$$
\psi_{V}(\xi, \tau)=\int_{\mathbf{R}^{\tau^{\prime}, \tau[}} v(x, N) F_{0}(I) \mathbf{1}_{C}(x) .
$$

Consider Riemann sums which, in the limit, give the left hand side of (12). By (12), we can remove all those terms containing $I[N]$ which are attached to points (or paths) $x$ which are discontinuous at some $t \in N$ without affecting, in the limit, the value of the Riemann sums.

But how will the overall limiting value of the Riemann sums be affected if we remove from the Riemann sums all those terms containing $I[N]$ which are attached to those $x$ which are discontinuous at any $t \in] \tau^{\prime}, \tau[$, a much larger set? 
Following the classical method of construction of Brownian motion, we adjust or modify the pre-measure $G_{0}$ in a way which leaves the values of the Riemann sums unaffected by this procedure, just as it is unaffected in (12).

Suppose $\left.t^{\prime}, t^{\prime \prime}, t^{\prime \prime \prime}, \ldots \in\right] \tau^{\prime}, \tau[$ are "close together". Then for the continuous $x$ of Brownian motion, the values $x\left(t^{\prime}\right), x\left(t^{\prime \prime}\right), x\left(t^{\prime \prime \prime}\right), \ldots$ will be "close together", - nearly equal but rarely equal. When we represent the $t$ 's by a system of Cartesian co-ordinates, such $x$ 's will be "fairly close" to a diagonal axis which is equi-angular to the $t^{\prime}, t^{\prime \prime}, t^{\prime \prime \prime}, \ldots$ axes. This is how the familiar jagged path picture of an everywhere continuous and nowhere differentiable element of Brownian motion translates into a point in a Cartesian co-ordinate scenario.

These properties of Brownian motion carry over into the Feynman setting. See Muldowney ([10, Propositions 47, p. 62, and 88, p. 103]); and Muldowney ([11, pp. 131-135]).

For those $x$ 's which are not close to the diagonal axis, differences between the values $x\left(t^{\prime}\right), x\left(t^{\prime \prime}\right), x\left(t^{\prime \prime \prime}\right) \ldots$ will be larger, and the terms of Riemann sum which are evaluated at such $x$ 's, when grouped together, will be subject to the cancellation effects described by Feynman - provided we are using a non-absolute method of integration such as Henstock's. They will contribute, in aggregate, little or nothing to the net value of their Riemann sum.

By (12) we know that this will work for $x$ which are removed from the Riemann sum for failing the test of continuity at $t \in N$. To ensure that it works for those $x$ which may, in addition, fail the test of continuity at $t \in] \tau^{\prime}, \tau\left[\backslash N\right.$, we modify $G_{0}$ in such a way that the latter terms get included in the Riemann sum in a different guise. That is the rationale for the following. Let

$$
F_{0}(y, M, J):= \begin{cases}\int_{J[M]} G_{0}(I[N]) \mathbf{1}_{C_{M}}(x) & \text { for } y \in C, \\ 0 & \text { for } y \notin C .\end{cases}
$$

Let

$$
F_{0}(J[M]):=\int_{\mathbf{R}^{] \tau^{\prime}, \tau[}} F_{0}(x, M, I), F_{0}(J(M)):=F_{0}(J[M]) .
$$

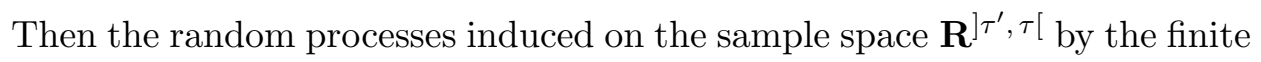
dimensional distribution $F_{0}(I)$ is a continuous modification of the process induced by $G_{0}(I)$. We shall prove that $F_{0}(I)$ corresponds to a random process with continuous sample paths, See also Muldowney [13, Theorem 5].

The Feynman volume or pre-measure $G_{0}(I)$ is $\mathrm{VBG}^{*}$ (i.e. has generalised bounded variation). That is, there exist sets $X_{k}$ in $\mathbf{R}^{] \tau^{\prime}, \tau[\text {, positive numbers }}$ $\alpha_{k}$, and a gauge $\gamma_{1}$ so that, for every $\mathcal{E}_{\gamma_{1}}$,

$$
\left(\mathcal{E}_{\gamma_{1}}\right) \sum\left|G_{0}(I)\right| \mathbf{1}_{X_{k}}(x)<\alpha_{k}, k=1,2, \ldots
$$


For proof, see Muldowney [10, Propositions 83, 84 and 85, pp. 97-98].

For ease of reference, we now gather together some of the results that have been examined in this section. Reviewing the notation, $g$ refers to the Gaussian kernel of the Feynman theory; the upper-case $G$ refers to integrated versions of $g$; the subscript $V$ specifies the potential function applied to the system; and the subscript 0 indicates the important $V=0$ case. $F$ designates the continuous modification of $G$.

Proposition 2. $\psi_{0}(\xi, \tau)=\int_{\mathbf{R}^{] \tau, \tau[}} G_{0}([N])$ exists and equals

$$
\left(\frac{\pi}{-\lambda}\left(\tau-\tau^{\prime}\right)\right)^{-1 / 2} \exp \frac{\lambda\left(\xi-\xi^{\prime}\right)^{2}}{\tau-\tau^{\prime}}
$$

$\int_{E} G_{0}(x, N, I)$ exists for every elementary set $E$.

Proposition 3. If $v(x, N) G_{0}(I)$ is integrable then $g_{V}(x, N, I)$ and $G_{V}(x, N, I)$ are integrable, and

$$
\psi_{V}(\xi, \tau)=\int_{\mathbf{R}^{] \tau, \tau[}} v(x, N) G_{0}(I)=\int_{\mathbf{R}^{] \tau, \tau[}} g_{V}(x, N, I)=\int_{\mathbf{R}^{] \tau, \tau[}} G_{V}(x, N, I) .
$$

Proposition 4. $g_{V}(x, N, I), G_{V}(x, N, I)$ are $\mathrm{VBG}^{*}$.

Proposition 5. For any cylinder set $J[M], F_{0}(J[M])=G_{0}(J[M])$.

Proof. This follows from (13) and Proposition 2.

Proposition 6. $F_{0}(x, N, I)$ is $\mathrm{VBG}^{*}$.

Proof. Follows from Proposition 5 since $G$ is $\mathrm{VBG}^{*}$.

Proposition 7. If $v(x, N) G_{0}$ is integrable then so is $v(x, N) F_{0}$, and

$$
\psi_{V}(\xi, \tau)=\int_{\mathbf{R}^{\mid \tau, \tau[}} v(x, N) G_{0}(I)=\int_{\mathbf{R}^{] \tau, \tau[}} v(x, N) F_{0}(I)=\int_{C} v(x, N) F_{0}(I) .
$$

Proof. Follows from (13) and (5).

Recalling the definition of $v(x)$ in (9), we prove a version of the FeynmanKac formula.

Proposition 8. If $V$ is continuous and $\psi_{V}(\xi, \tau)$ exists, then

$$
\psi_{V}(\xi, \tau)=\int_{\mathbf{R}^{\tau, \tau[}} \mathbf{1}_{C}(x) v(x) F_{0}(I)=\int_{C} v(x) F_{0}(I) .
$$


Proof. Since $F_{0}$ is $\mathrm{VBG}^{*}$; we can choose $\gamma_{1}$ in (14) so that for each $x \in X_{j}$, $N \supset L_{A}(x)$ implies $|v(x, N)-v(x)|<\varepsilon 2^{-j} \alpha_{j}$. Then for each $\mathcal{E}_{\gamma_{1}}$,

$$
\begin{aligned}
\left|\left(\mathcal{E}_{\gamma_{1}}\right) \sum(v(x, N)-v(x)) F_{0}(I)\right| & \leq\left(\mathcal{E}_{\gamma_{1}} \sum|v(x, N)-v(x)|\left|F_{0}(I)\right|\right. \\
& =\sum_{j=1}^{\infty} \sum_{x \in X_{j}}|v(x, N)-v(x)|\left|F_{0}(I)\right| \\
& <\sum_{j=1}^{\infty} \varepsilon 2^{-j} \alpha_{j}^{-1} \alpha_{j}=\varepsilon .
\end{aligned}
$$

The result follows by Proposition 7 .

The corresponding result for Brownian motion $(\lambda=-1)$ is (4) above. If we were to use the notation of (4) here, we would write

$$
\psi_{V}(\xi, \tau)=\int_{C(] \tau^{\prime}, \tau[)}\left(\int_{\tau^{\prime}}^{\tau} \exp (\lambda V(x(t))) d t\right) d F_{0} .
$$

However the $d F_{0}$ notation is unsuitable in our terms, since it implies $F_{0}$ is a measure, which it is not.

\section{Feynman's limit}

We have defined $\psi_{V}(\xi, \tau)$ as a Henstock integral over the function space, and, having done so, are interested in establishing the conditions for which Feynman's limit (2) is, in our terms, valid ${ }^{3}$. In other words, we want to show that the existence of Feynman's limit implies the existence of the Henstock integral (11) and the equality of the two.

With $\tau_{j}=\tau+j 2^{-q}\left(\tau-\tau^{\prime}\right), j=1, \ldots, m-1$, where $m=2^{q}$, let

$$
M:=\left\{\tau_{1}, \ldots, \tau_{m-1}\right\} .
$$

For $y=\left(y_{1}, \ldots, y_{m-1}\right) \in \mathbf{R}^{m-1}, y_{j}=y\left(\tau_{j}\right)$ for $1 \leq j \leq m-1, y_{0}=\xi^{\prime}$ and $y_{m}=\xi$, let $g_{V}^{(m)}(y)$ denote

$\exp \left(\lambda \sum_{j=1}^{m} \frac{1}{2} \frac{\left(y_{j}-y_{j-1}\right)^{2}}{\tau_{j}-\tau_{j-1}}-V\left(y_{j-1}\right)\left(\tau_{j}-\tau_{j-1}\right)\right) \prod_{j=1}^{m}\left(\frac{2 \pi}{-\lambda}\left(\tau_{j}-\tau_{j-1}\right)\right)^{-1 / 2}$.

In effect, Feynman defined the state function $\psi_{V}(\xi, \tau)$ to be the following limit:

$$
\lim _{m \rightarrow \infty} \int_{\mathbf{R}^{m-1}} g_{V}^{(m)}(y) d y
$$

\footnotetext{
${ }^{3}$ It is obviously valid when the potential $V$ is identically zero (the free particle case). See Muldowney [10, Proposition 68, page 84].
} 
(15) is (2) in an alternative notation. Note that Feynman used $\tau_{j}=\tau_{j-1}+$ $m^{-1}\left(\tau-\tau^{\prime}\right)$ (see (1) and (2) above), whereas we find it convenient to use $m=2^{q}$ and $\tau_{j}=\tau_{j-1}+2^{-q}\left(\tau-\tau^{\prime}\right)$, so we will be dealing with a subsequence of Feynman's sequence of finite dimensional integrals.

Proposition 9. If $V$ is continuous and $\int_{\mathbf{R}^{m-1}} g_{V}^{(m)}(y) d y$ exists, and equals $f_{m}$, then

$$
\int_{\mathbf{R}^{] \tau^{\prime}, \tau[}} v(x ; M) G_{0}(I)
$$

exists and equals $f_{m}$. So

$$
\int_{\mathbf{R}^{\mid \tau^{\prime}, \tau[}} v(x ; M) G_{0}(I)=f_{m},
$$

and there exists $\gamma_{2}$ so that, for every $\mathcal{E}_{\gamma_{2}}$,

$$
\left|\left(\mathcal{E}_{\gamma_{2}}\right) \sum v(x ; M) G_{0}(I)-f_{m}\right|<\varepsilon .
$$

Proof. Follows from Muldowney [10, Proposition 69, p. 87].

Suppose $h_{m}\left(y^{(m)}\right)$ is defined for $y^{(m)} \in \mathbf{R}^{M}$. We say that $h_{m}$ is uniformly integrable to $H_{m}, m=1,2,3, \ldots$, if, given $\varepsilon>0$, there exist positive functions $\left\{\delta_{0}\right\}$ defined for $y^{(m)} \in \mathbf{R}^{M}$, with $\delta_{0}\left(y^{(m)}\right)=\delta_{0}\left(y^{(m+1)}\right)$, $m=1,2,3, \ldots$, such that, for all $\delta_{0}$-fine divisions $\mathcal{E}_{\delta_{0}}$ of $\mathbf{R}^{M}$,

$$
\left|\left(\mathcal{E}_{\delta_{0}}\right) \sum h_{m}\left(y^{(m)}\right)\right| I(M)\left|-H_{m}\right|<\varepsilon .
$$

Proposition 10. If $V$ is continuous, $G_{V}^{(m)}(y)$ is uniformly integrable in $\mathbf{R}^{M}, m=2,3, \ldots$, and $f_{m}=\int_{\mathbf{R}^{M}} G_{V}^{(m)}(y) d y$ converges to a limit $f$ as $m \rightarrow \infty$, then $\psi_{V}(\xi, \tau),=\int_{\mathbf{R}^{] \tau^{\prime}, \tau[}} v(x, N) G_{0}(I)$, exists and equals $f$.

Proof. The first of Henstock's criteria (6) is the one which gives this result. Let $\varepsilon>0$ be given. We need to define a gauge $\gamma$ and integers $p(x)$ so that for $m(x)>p(x)$ and all $\mathcal{E}_{\gamma}$,

$$
\left|\left(\mathcal{E}_{\gamma}\right) \sum v_{m(x)}(x) F_{0}(I)-f\right|
$$

is of order $\varepsilon$. Choose $s_{0}$ so that $s>s_{0}$ implies $\left|f_{s}-f\right|<\varepsilon$. For each $x \in X_{k}$, choose $p(x)>s_{0}$ so that $m(x)>p(x)$ implies

$$
\left|v_{m(x)}(x)-v(x)\right|<\varepsilon \alpha_{k}^{-1} 2^{-k} .
$$

Then

$$
\left|v_{m(x)}(x) F_{0}(I)-v(x) F_{0}(I)\right|<\varepsilon \alpha_{k}^{-1} 2^{-k}\left|F_{0}(I)\right|,
$$


so, taking $\gamma_{0} \prec \gamma_{1}$ in (14) above, and

$$
\beta(x, N, I)=\left|F_{0}(I)\right| \alpha_{k}^{-1} 2^{-k},
$$

we have

$$
\left(\mathcal{E}_{\gamma_{0}}\right) \sum \beta(x, N, I)<\sum_{k=1}^{\infty} 2^{-k}=1,
$$

so $v_{m}(x) F_{0}(I)$ converges boundedly (see (5)) to $v(x) F_{0}(I)$. Choose $\gamma \prec$ $\gamma_{0} \wedge \gamma_{2}$ (where the gauge $\gamma_{2}$ is defined in (17) above), and let $\gamma=\left(\delta, L_{A}\right)$ satisfy, in addition,

$$
\begin{aligned}
A & \supseteq \cup_{r=0}^{\infty}\left\{\tau_{j}: j=1, \ldots, r-1\right\}, \\
L_{A}(x) & \supseteq\left\{\tau_{1}, \ldots, \tau_{p(x)}\right\}, \\
\delta(x) & \leq \delta_{0}\left(x\left(\tau_{j}\right)\right),
\end{aligned}
$$

where $\delta_{0}$ is the gauge of uniform integrability of $G_{V}^{(m)}$. Given a division $\mathcal{E}_{\gamma}$ and a Riemann sum $\left(\mathcal{E}_{\gamma}\right) \sum v_{m(x)}(x) F_{0}(I)$ with $m(x)>p(x)$, let

$$
m=\max \left\{m(x):(x, I[N]) \in \mathcal{E}_{\gamma}\right\}, M=\left\{\tau_{1}, \ldots, \tau_{m-1}\right\} .
$$

Then

$$
\left|\left(\mathcal{E}_{\gamma}\right) \sum v_{m(x)}(x) F_{0}(I)-f\right| \leq a+b+c+d,
$$

where

$$
\begin{aligned}
a & =\left|\left(\mathcal{E}_{\gamma}\right) \sum v_{m(x)}(x) F_{0}(I)-\left(\mathcal{E}_{\gamma}\right) \sum v_{m}(x) F_{0}(I)\right|, \\
b & =\left|\left(\mathcal{E}_{\gamma}\right) \sum v_{m}(x) F_{0}(I)-\left(\mathcal{E}_{\gamma}\right) \sum v(x ; M) F_{0}(I)\right|, \\
c & =\left|\left(\mathcal{E}_{\gamma}\right) \sum v(x ; M) F_{0}(I)-f_{m}\right|, \\
d & =\left|f_{m}-f\right| .
\end{aligned}
$$

We have

$$
\begin{aligned}
a & <2 \varepsilon \text { since } m>p(x) \text { and } m(x)>p(x), \\
b & =0, \\
c & <\varepsilon, \text { by uniform integrability, and } \\
d & <\varepsilon .
\end{aligned}
$$

This completes the proof.

Essentially, this proposition establishes that Feynman's definition ((2) or (15)) of the state function coincides with the definition (11) provided by the Henstock theory, under conditions of continuity and smoothness of $V$. For instance, if $V$ is constant the hypotheses of the proposition are valid, and the conclusion of the proposition is confirmed in this simple case by Proposition 68 of Muldowney [10, p. 84]. 
It also gives conditions of a finite dimensional character for the existence of Feynman integrals, giving access to the computation and evaluation of such integrals. See, for example, Montroll [9] and Gelfand and Yaglom [3].

As in the following section, simpler and more familiar methods of proof will give the results for the special case $\lambda=-1$ (Brownian motion). See Muldowney [10, Proposition 53, p. 67, and Proposition 57, p. 70].

\section{Schrödinger's equation}

We wish to prove that $\psi_{V}(\xi, \tau)$ satisfies Schrödinger's equation. That is,

$$
\frac{\partial \psi_{V}}{\partial \tau}-\frac{\lambda}{2} \frac{\partial^{2} \psi_{V}}{\partial \xi^{2}}+\lambda V(\xi) \psi_{V}=0
$$

This is clearly true for some simple classes of $V$. See Muldowney [10, Proposition 68, p. 84, and pp. 100-103] .

What is involved in the various partial derivatives? We have

$$
\psi_{V}=\int_{\mathbf{R}^{\left[\tau^{\prime}, \tau[\right.}} g_{V}(x, N, I), \frac{\partial \psi_{V}}{\partial \tau}=\frac{\partial}{\partial \tau} \int_{\mathbf{R}^{\left[\tau^{\prime}, \tau[\right.}} g_{V}(x, N, I),
$$

where the integrand

$$
g_{V}(x, N, I)=g_{V}(x, N, I ; \xi, \tau)
$$

depends on $\xi$ and $\tau$. We demonstrate below that $g_{V}$ satisfies

$$
\frac{\partial g_{V}}{\partial \tau}-\frac{\lambda}{2} \frac{\partial^{2} g_{V}}{\partial \xi^{2}}+\lambda V\left(x_{n-1}\right) g_{V}=0
$$

with $x_{n-1}$ approaching $\xi$, and we may be able to use some theorem on differentiation under the integral sign. But the space $\mathbf{R}^{]} \tau^{\prime}, \tau$, over which we are integrating, also varies when we vary $\tau$. Methods such as dominated convergence or Henstock's criteria cannot be applied straightforwardly in such a situation.

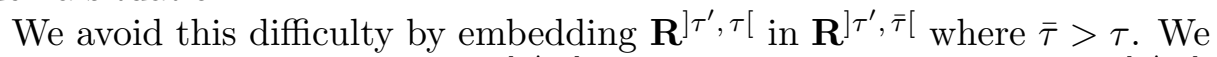
let $\bar{x}, \bar{N}, \bar{I}$ denote objects in $\mathbf{R}^{] \tau^{\prime}, \bar{\tau}}$, corresponding to $x, N, I$ in $\mathbf{R}^{] \tau^{\prime}, \tau[\text {, }}$ where $N=\left\{t_{1}, \ldots, t_{n-1}\right\}$ with $t_{n}=\tau, \bar{N}=\left\{t_{1}, \ldots, t_{n-1}, t_{n}, \ldots, t_{m-1}\right\}$

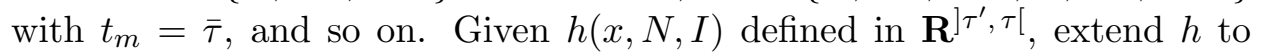
$\mathbf{R}^{] \tau^{\prime}, \bar{\tau}[\text { as follows. }}$

$$
h(\bar{x}, \bar{N}, \bar{I})=\left\{\begin{array}{lll}
0 & \text { if } t \in \bar{N} \backslash N & \text { and } \bar{x}(t) \neq 0 \\
h(x, N, I) & \text { if } t \in \bar{N} \backslash N & \text { and } x(t)=0 .
\end{array}\right.
$$

In the analysis below, we assume such an embedding has been performed, so limits involving $\tau$ can be taken independently of the space in which integration is performed. 
Recall that $g_{V}(x, N, I)$ (or $\left.g_{V}(x, N, I ; \xi, \tau)\right)$ denotes

$\exp \left(\lambda \sum_{j=1}^{n}\left(\frac{1}{2} \frac{\left(x_{j}-x_{j-1}\right)^{2}}{t_{j}-t_{j-1}}-V\left(x_{j-1}\right)\left(t_{j}-t_{j-1}\right)\right)\right) \prod_{j=1}^{n}\left(\frac{2 \pi}{-\lambda}\left(t_{j}-t_{j-1}\right)\right)^{-1 / 2}$

where

$$
x_{0}=\xi^{\prime}, x_{n}=\xi, t_{0}=\tau^{\prime}, t_{n}=\tau .
$$

By differentiation,

$$
\begin{aligned}
\frac{\partial g_{V}}{\partial \tau} & =-\frac{1}{2}\left(\tau-t_{n-1}\right)^{-1} g_{V}-\left(\frac{\lambda}{2}\left(\frac{\xi-x_{n-1}}{\tau-t_{n-1}}\right)^{2}+\lambda V\left(x_{n-1}\right)\right) g_{V} \\
\frac{\partial g_{V}}{\partial \xi} & =\lambda \frac{\xi-x_{n-1}}{\tau-t_{n-1}} g_{V} \\
\frac{\partial^{2} g_{V}}{\partial \xi^{2}} & =\frac{\lambda}{\tau-t_{n-1}} g_{V}-\left(\frac{\xi-x_{n-1}}{\tau-t_{n-1}}\right)^{2} g_{V} .
\end{aligned}
$$

Thus

Now recall that

$$
\frac{\partial g_{V}}{\partial \tau}-\frac{\lambda}{2} \frac{\partial^{2} g_{V}}{\partial \xi^{2}}+\lambda V\left(x_{n-1}\right) g_{V}=0
$$

$$
\psi_{V}=\int_{\mathbf{R}^{\prime \tau^{\prime}}, \tau[} g_{V}(x, N, I[N])=\int_{\mathbf{R}^{] \tau^{\prime}, \bar{\tau}[}} g_{V}(\bar{x}, \bar{N}, \bar{I}[\bar{N}]) .
$$

Then, provided we can reverse the order of integration and differentiation, we get

$$
\frac{\partial \psi_{V}}{\partial \tau}-\frac{\lambda}{2} \frac{\partial^{2} \psi_{V}}{\partial \xi^{2}}+\lambda V(\xi) \psi_{V}=0
$$

since the sample paths are continuous and $x_{n-1} \rightarrow \xi, V\left(x_{n-1}\right) \rightarrow V(\xi)$ as $n \rightarrow \infty$ provided $V$ is continuous at $\xi$. In the case $\lambda=-1$, we can use the dominated convergence theorem to justify changing the order of integration and differentiation, and the equation is a diffusion equation for Brownian motion. See Muldowney [10, Proposition 57, p. 70], also [10, pp. 101-103]. The method used there is similar to that used by Kac [8].

If $\lambda$ has an imaginary component, or $\lambda=\iota$, such a simple approach is generally impossible, and we must use Henstock's criteria for taking limits under the integral sign. For brevity, we make some notational short cuts. Given $h(\xi, \tau)$, let

$$
D_{a b c} h(\xi, \tau):=\frac{1}{a} h_{a}(\xi, \tau)-\frac{\lambda}{b c} h_{b c}(\xi, \tau)
$$

where

$$
\begin{aligned}
h_{a}(\xi, \tau) & =h(\xi, \tau+a) \\
h_{b c}(\xi, \tau) & =h(\xi+b+c, \tau)-h(\xi+b, \tau)-h(\xi+c, \tau)+h(\xi, \tau)
\end{aligned}
$$


for non-zero real numbers $a, b, c$. Then

$$
\lim _{a, b, c \rightarrow 0} D_{a b c} h(\xi, \tau)
$$

exists and equals

$$
\frac{\partial h}{\partial \tau}-\frac{\lambda}{2} \frac{\partial^{2} h}{\partial \xi^{2}}
$$

if and only if the partial derivatives

$$
\frac{\partial h}{\partial \tau}, \frac{\partial^{2} h}{\partial \xi^{2}}
$$

exist. From the differentiation above we have

$$
\lim _{a, b, c \rightarrow 0} D_{a b c} g_{V}=-\lambda V\left(x_{n-1}\right) g_{V},
$$

and we assume that $g_{V}(\bar{x}, \bar{N}, \bar{I})$ is integrable in $\mathbf{R}^{] \tau^{\prime}, \bar{\tau}[}$ for a range of values of $\xi$ and $\tau$.

Then, provided $V$ is continuous at $\xi, V\left(x_{n-1}\right) g_{V}$ is integrable in $\mathbf{R}^{] \tau^{\prime}, \bar{\tau}}$, and $\int V\left(x_{n-1}\right) g_{V}=V(\xi) \psi_{V}$. See Muldowney [10, Proposition 86, p. 98], for a proof of this. Thus, provided $V$ is continuous at $\xi$,

$$
\int_{\mathbf{R}^{] \tau^{\prime}, \bar{\tau}[}} \lim _{a, b, c \rightarrow 0} D_{a b c} g_{V}
$$

exists and equals $-\lambda V(\xi) \psi_{V}$.

By assumption, $\int D_{a b c} g_{V},=D_{a b c} \int g_{V}$, exists for a range of $a, b, c$. The questions we must resolve are:

1. Does $\lim _{a, b, c \rightarrow 0} D_{a b c} \int g_{V}$ exist? That is, do the partial derivatives

$$
\frac{\partial \psi_{V}}{\partial \tau}, \frac{\partial^{2} \psi_{V}}{\partial \xi^{2}}
$$

exist?

2. Is $\lim _{a, b, c \rightarrow 0} D_{a b c} \int g_{V}$ (when it exists) equal to $\int \lim _{a, b, c \rightarrow 0} D_{a b c} g_{V}$ ? In other words, does the following hold?

$$
\left(\frac{\partial}{\partial \tau}-\frac{\lambda}{2} \frac{\partial^{2}}{\partial \xi^{2}}\right) \psi_{V}=-\lambda V(\xi) \psi_{V}
$$

Proposition 11. If $V$ is continuous at $\xi$, and provided we have $\xi_{0}$ and $\tau_{0}$ with $g_{V}(\bar{x}, \bar{N}, \bar{I})$ integrable in $\mathbf{R}^{\tau^{\prime}, \bar{\tau}[}$ for each $\xi$ and $\tau$ in a neighbourhood of $\xi_{0}$ and $\tau_{0}$, then

$$
\frac{\partial \psi_{V}}{\partial \tau}-\frac{\lambda}{2} \frac{\partial^{2} \psi_{V}}{\partial \xi^{2}}+\lambda V(\xi) \psi_{V}=0
$$


Proof. In this case, the second of Henstock's criteria (7) is the one we need. First we consider the convergence of $D_{a b c} \int g_{V},\left(=\int D_{a b c} g_{V}\right)$ as $a, b, c \rightarrow 0$. Let $\varepsilon>0$ and $a, b, c$ (with $\tau^{\prime}-\tau<a<\bar{\tau}-\tau$ and $\tau$ in the appropriate neighbourhood of $\tau_{0}$ ) be given. Let $\mathcal{E}$ and $\mathcal{D}$ denote divisions of $\mathbf{R}^{] \tau^{\prime}, \bar{\tau}[}$, and consider

$$
D_{a b c}(\mathcal{E}) \sum g_{V}(\xi, \tau)-D_{a b c}(\mathcal{D}) \sum g_{V}(\xi, \tau)
$$

This expression can be re-arranged so that it consists of

$$
\frac{1}{a}(\mathcal{E}) \sum g_{V}(\xi, \tau+a)-\frac{1}{a}(\mathcal{D}) \sum g_{V}(\xi, \tau+a)
$$

and five similar terms. By the assumed integrability of $g_{V}$, a gauge $\gamma_{1}$ can be chosen so that

$$
\left|\frac{1}{a}\left(\mathcal{E}_{\gamma_{1}}\right) \sum g_{V}(\xi, \tau+a)-\frac{1}{a}\left(\mathcal{D}_{\gamma_{1}}\right) \sum g_{V}(\xi, \tau+a)\right|<\varepsilon .
$$

Similarly define

$$
\gamma_{2}, \ldots, \gamma_{6}
$$

for the other five terms, respectively. Now let $\gamma$ satisfy

$$
\gamma \prec \gamma_{1} \wedge \gamma_{2} \wedge \ldots \wedge \gamma_{6} \text {. }
$$

So

$$
\left|D_{a b c}\left(\mathcal{E}_{\gamma}\right) \sum g_{V}(\xi, \tau)-D_{a b c}\left(\mathcal{D}_{\gamma}\right) \sum g_{V}(\xi, \tau)\right|<6 \varepsilon
$$

Thus

$$
\frac{\partial \psi_{V}}{\partial \tau}, \frac{\partial^{2} \psi_{V}}{\partial \xi^{2}}
$$

exist whenever $g_{V}$ is integrable for a suitable range of $\xi, \tau$. Now consider the equality of $\lim _{a, b, c \rightarrow 0} \int g_{V}$ and $\int \lim _{a, b, c \rightarrow 0} D_{a b c} g_{V}$. We have

$$
\left(\frac{\partial}{\partial \tau}-\lambda \frac{\partial^{2}}{\partial \xi^{2}}\right) \sum g_{V}(\xi, \tau)=\sum-V\left(x_{n-1}\right) g_{V}(\xi, \tau) .
$$

$\gamma$ can be chosen so that

$$
\left|\left(\mathcal{E}_{\gamma}\right) \sum\left(-V\left(x_{n-1}\right) g_{V}\right)-\left(-V(\xi) \psi_{V}\right)\right|<\varepsilon,
$$

and, given $\varepsilon$, we can find $a_{0}, b_{0}, c_{0}>0$ so that

$$
|a|,|b|,|c|<a_{0}, b_{0}, c_{0}
$$

imply

So

$$
\left|D_{a b c}\left(\mathcal{E}_{\gamma}\right) \sum g_{V}-\left(\mathcal{E}_{\gamma}\right) \sum\left(-V\left(x_{n-1}\right) g_{V}\right)\right|<\varepsilon
$$

$$
\left|D_{a b c}\left(\mathcal{E}_{\gamma}\right) \sum g_{V}-V(\xi) \psi_{V}\right|<2 \varepsilon .
$$

Therefore the requirements of Henstock's criteria are satisfied and the proposition is proved. 


\section{Conclusion}

To sum up, we have taken Feynman's concept of probability amplitude and used it to define the state function of quantum mechanics, in a manner which is consistent with, and is an extension of, the probabilistic analysis of Brownian motion.

We have established conditions for which Feynman's limit of finite dimensional integrals gives the state function as we have defined it.

This solution to the Feynman problem depends on Henstock's theory of non-absolute integration in function spaces, and on Henstock's criteria for taking limits under the integral sign.

\section{References}

[1] Dirac, P. A. M., The Principles of Quantum Mechanics, Clarendon Press, Oxford, 1935.

[2] Feynman, R. P., Space-time approach to non-relativistic quantum mechanics, Rev. Modern Phys. 20(2) (1948), 367-387.

[3] Gelfand, I. M., and Yaglom, A. M., Integration in function spaces, J. Math. Phys. 1 (1960), 48-69.

[4] Henstock, R., Linear Analysis, Butterworths, London, 1968.

[5] Henstock, R., Generalised integrals of vector-valued functions, Proc. London Math. Soc. (3) 19 (1969), 509-536.

[6] Henstock, R., Integration in product spaces, including Wiener and Feynman integration, Proc. London Math. Soc. 93) 27 (1973), 317-344.

[7] Henstock, R., The General Theory of Integration, Clarendon Press, Oxford, 1991.

[8] Kac, M., Probability and Related Topics in the Physical Sciences, Interscience, New York, 1957.

[9] Montroll, E. W., Markoff chains, Wiener integrals and quantum theory, Comm. Pure Appl. Math. 5 (1952), 415-453.

[10] Muldowney, P., A General Theory of Integration in Function Spaces, Pitman Res. Notes Math. Ser., Longman, Harlow, 1987.

[11] Muldowney, P., Infinite-dimensional generalised Riemann integrals, in "New Integrals", Springer-Verlag, Berlin, 1990, 131-135.

[12] Muldowney, P., Introduction to Feynman integration, J. Math. Study 27(1) (1994), 127-132.

[13] Muldowney, P., Topics in probability using generalised Riemann integration, Proc. Roy. Irish Acad. Sect. A 99(1) (1999), 39-50.

Pat Muldowney

Magee College

UNIVERSITY OF ULSTER

NoRThland Road

DERRY BT 48 7JL

North IRELAND

E-MAIL: P.MuldOWNEY@Ulst.AC.UK 